\title{
Differential expression and activity of matrix metalloproteinases 2 and 9 in canine early placenta
}

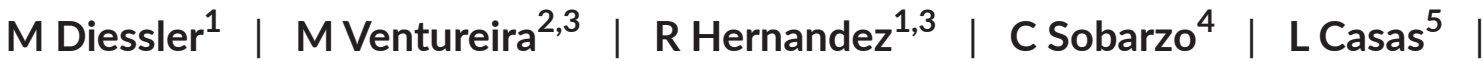 \\ C Barbeito $^{1,3} \mid$ E Cebral $^{2,3}$
}

${ }^{1}$ Laboratorio de Histología y Embriología Descriptiva, Experimental y Comparada (LHYEDEC), Facultad de Ciencias

Veterinarias, Universidad Nacional de La Plata (UNLP), La Plata, Argentina

${ }^{2}$ Laboratorio de Reproducción y Fisiopatología Materno-

Embrionaria, Instituto de Fisiología, Biología Molecular y Neurociencias (IFIBYNE), Universidad de Buenos

Aires (UBA)- Comisión Nacional de Investigaciones Científicas (CONICET), Facultad de Ciencias Exactas y Naturales (FCEyN)-UBA, Buenos Aires, Argentina

${ }^{3}$ Instituto de Investigaciones Biomédicas (INBIOMED)-UBA/CONICET, Facultad de Medicina, UBA, Buenos Aires, Argentina

${ }^{4}$ Facultad de Medicina, Instituto de Investigaciones Biomédicas (INBIOMED)UBA/CONICET, UBA, Buenos Aires, Argentina

${ }^{5}$ Small Animal Private Practice, La Plata, Argentina

\section{Correspondence}

Claudio Barbeito, Laboratorio de Histología y Embriología Descriptiva, Experimental y Comparada (LHYEDEC), Facultad de Ciencias Veterinarias, Universidad Nacional de La Plata (UNLP), 60 y 118, La Plata, Argentina. Email: barbeito@fcv.unlp.edu.ar

\section{Contents}

The zonary and endotheliochorial dog placenta is the most invasive placenta of carnivores. The importance of matrix metalloproteinases (MMP) in placenta invasiveness has been determined in several mammals including species with haemochorial, epitheliochorial and endotheliochorial placentation. Regarding the latter, the expression of MMP enzymes has been studied in the cat and the mature canine placenta. The aim of this study was to analyse the expression and activity of MMP-2 and MMP-9 in the early dog placenta. Placentae from 18 to 30 days of pregnancy were collected from four bitches. Two placentae from each bitch were analysed. Placental tissue from one uterine horn was fixed in formaldehyde for immunohistochemistry, while marginal haematoma, labyrinth, non-implantative and implantative endometrium from the contralateral horn were immediately frozen in dry ice for the analysis of MMP expression (Western blot [WB]) and activity (zymography). MMP-2 and MMP-9 were evidenced in the labyrinth, maternal glands and marginal haematoma; this finding was directly correlated with levels of MMP expression by WB, and with the activity of MMP-2, mainly in the haematoma (the area of major remodelling of tissues). Thus, although MMP-9 is well expressed in the early canine placenta, it is not active. Given the important role of MMPs for invasiveness, maternal-foetal angiogenesis and the establishment of a correct foetal nutrition, the results are consistent with the findings in other species in which the MMP-2 activation precedes the MMP-9 one in early placentation.

\section{1 | INTRODUCTION}

The placenta presents marked differences amongst eutherians. Most carnivores, except for the hyena, develop an endotheliochorial and zonary type of placenta. Haemochorial type is the most invasive placenta and develops in most primates, rodents and hyenas, whereas the epitheliochorial type is the less invasive placenta and characterizes, for example swine and equine placentation. By virtue of the loss of most endometrial structures during placentation, except for the vessels, the endotheliochorial placenta is considered of intermediate invasiveness

C. Barbeito and E. Cebral contributed equally to the design and leading of this work.
(Enders \& Carter, 2012a,b; Pfarrer, Winther, Leiser, \& Dantzer, 1999; Wooding \& Burton, 2008). However, close contact between the trophoblast (TB) cells and stagnant maternal blood does occur in endotheliochorial placentae in some haematophagous areas where, unlike the labyrinth, maternal vessels are degraded. In the dog, these haematophagous placental areas constitute the marginal haematoma, located peripherally to the placental belt (Aralla, Groppetti, Caldarini, Cremonesi, \& Arrighi, 2013; Miglino et al., 2006).

The endotheliochorial placenta of the dog is composed by different regions: the chorioallantoic membrane (CAM), the labyrinthine, junctional and deep glandular zones and the marginal haematomas (Wooding \& Burton, 2008) (Fig. 1). The CAM is formed by the allantoic 


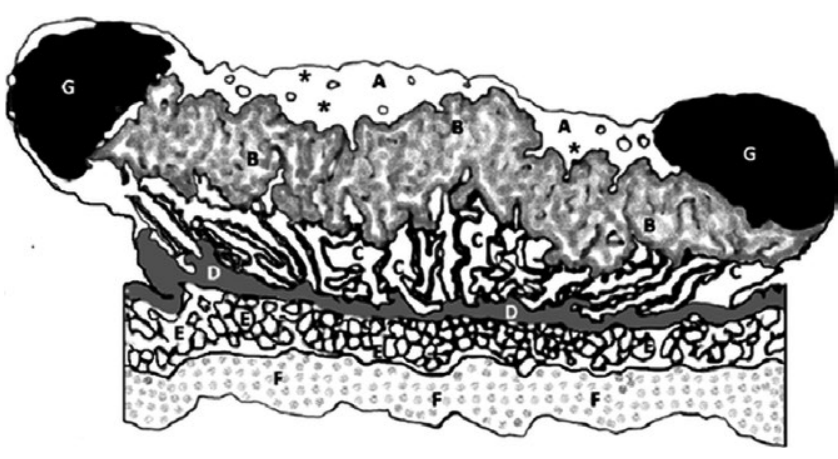

FIGURE 1 Regions of the endotheliochorial placenta of the dog. A. Chorioallantoic membrane with mesenchyme $\left({ }^{*}\right)$. B. Labyrinth. C. Superficial glands forming the spongy zone. D. Endometrial stroma. E. Deep glands. F. Myometrium. G. Marginal haematomas

endoderm, the mesenchyme of both allantoic and chorial origins and the TB covering the chorion. Chorioallantoic villi interdigitates with endometrium structures to form the labyrinth zone, where TB differentiated in cyto- and syncytiotrophoblast is observed. The junctional zone (the releasing zone at partum) exhibits superficial glands forming the "spongy zone".

The length of the normal canine gestation is approximately two months, or 65 days from the luteinizing hormone surge (VerstegenOnclin \& Verstegen, 2008). A placental labyrinth with cytotrophoblast and syncytiotrophoblast differentiation is already formed as soon as day 18 of gestation, and the organ continues its development during the first half of pregnancy until it is considered a mature placenta (Aralla et al., 2013). Marginal haematomas are well developed and green by 22-25 days of pregnancy (Miglino et al., 2006). Growth of the labyrinth depends upon remodelling of the maternal connective tissue and angiogenic stimuli.

Canine placentation is more invasive than that of the cat, despite their similarities. Absent or scarce decidual vimentin-positive cells, well described in cat but unsteadily identified in the dog, might partially explain its higher invasiveness (Barbeito, Fernández, Gimeno, \& Portiansky, 2004; Fernández, Barbeito, Portiansky, \& Gimeno, 2000; Fernández et al., 2014; Kautz et al., 2015; Walter \& Schönkypl, 2006). Furthermore, several cellular and matrix components participate in the invasive function of the early canine placenta. Recently, a role of galectins regarding invasiveness was proposed (Conrad et al., 2016). Also, endothelium of foetal and maternal vessels co-expresses VEGFA and VEGFR1, being involved both in angiogenic cascade and in cell signalling leading to the invasion process (Gram et al. 2015; Wooding \& Burton, 2008). Serine, cysteine and matrix metalloproteinases (MMPs) participate in remodelling uterine extracellular matrix (ECM) components through the degradation of gelatin, collagens, laminin, fibronectin and the establishment of vascular and anchoring links between foetus and mother. These coordinated and regulated actions of extracellular proteolytic enzymes require a fine balance between activated proteases and their tissue inhibitors (TIMPs) (Cross, Werb, \& Fisher, 1994; Sternlicht \& Werb, 2001).

Matrix metalloproteinasess are a group of zinc proteases with a similar domain structure, produced in a intracellular latent form and secreted as inactive precursors (pro-enzymes). Protease interactions exhibit considerable functional redundancy to ensure maternal and foetal connections. MMP-2 (gelatinase A) and MMP-9 (gelatinase B) share a similar structure and have a hydrolytic action on collagen type IV, which allows separation of cells from their basal lamina and, therefore, phenotype transformation (Teesalu, Masson, Basset, Blasi, \& Talarico, 1999). MMP-2 and MMP-9 functions have been related to embryo implantation (Novaro et al., 2002). Most studies have been performed in haemochorial placentae, which possess the highest degree of invasiveness (Li, Hou, Zhang, \& Zhang, 2014; Staun-Ram, Goldman, Gabarin, \& Shalev, 2004; Teesalu et al., 1999; Wang, Li, Shao, \& Zhu, 2001; Xu et al., 2000). Moreover, the presence and activity of MMP-2 and MMP-9 were determined in species developing epitheliochorial (Vagnoni, Ginther, \& Lunn, 1995) and synepitheliochorial placentae (Riley, Webb, Leask, McCaig, \& Howe, 2000; Takagi, Yamamoto, Ohtani, \& Miyamoto, 2007; Uekita et al., 2004; Vagnoni, Zheng, \& Magness, 1998; Walter \& Boos, 2001). Concerning feline endotheliochorial placentae, the expression of MMP-2 was reported, while no specific immunostaining for MMP-9 was evidenced (Walter \& Schönkypl, 2006). In dogs, MMP-2 was detected in the mature placenta in one study, mostly in vascular structures, while little amounts of MMP-9 were evidenced in the junctional zone (Beceriklisoy et al., 2007). Moreover, Kutzler et al. found activity of both enzymes in cultured canine TB cells of term placenta (Kutzler, Sahlfeld, \& Fellows, 2012).

Therefore, considering the pivotal role of MMP-2 and MMP-9 in invasiveness during early placental development, we performed this work to analyse the expression, localization, distribution and activity of these both enzymes in the early canine placenta.

\section{2 | MATERIALS AND METHODS}

\subsection{Canine placental tissue collection}

The dog (Canis lupus familiaris) specimens for this study were obtained with owners' approval, following elective pregnancy termination by ovariohysterectomy, at the Universidad Nacional de La Plata. One horn from each uterus was fixed by immediate immersion in $4 \%$ buffered formaldehyde for $24 \mathrm{hr}$, while the contralateral horn was dissected on dry ice and then stored at $-70^{\circ} \mathrm{C}$ for molecular studies.

In order to study MMPs expression in the early canine placentae, and lacking the exact day of the LH surge in the bitches, we took into account various macroscopical and microscopical features to determine which of the samples corresponded to the first half of the pregnancy. Therefore, samples with crown-rump embryo length lesser than $30 \mathrm{~mm}$, in tail bud or limb bud stages and lacking noticeable digital grooves (Evans \& Sack, 1973; Pieri et al., 2015) were chosen. Four pregnancies matched these criteria. In those selected specimens, embryo red cells exhibited characteristics of pre-liver erythropoiesis stages (yolk sac or aorta/gonad/mesonephros origin): nucleation, cell size several times larger than mature erythrocytes, cytoplasmic basophilia/amphophilia (McGrath \& Palis, 2005; Nothdurft et al., 1984). Histological aspect of the labyrinth and the glandular chambers from 
the spongy zone also confirmed the right inclusion of those samples in the study, according to previous descriptions (Aralla et al., 2013; Barrau, Abel, Torbit, \& Tietz, 1975).

Samples from two placentae of each of the four cases selected were processed for immunohistochemistry (IHC), Western blot (WB) and zymography (Zg).

For WB and $\mathrm{Zg}$, samples from the labyrinth (Lab), the nonimplantative (Non-Impl) and implantative endometrium (Impl-End) of each placentae were processed. Placentae from three of the four cases had marginal haematomas (Hem), also processed by means of WB and Zg.

\subsection{Immunohistochemistry}

Immunohistochemistry was performed to detect MMP-2 and MMP-9 expression in different zones of the early canine placenta. After formol fixation for $24 \mathrm{hr}$, tissues were deparaffinized and rehydrated through graded series of ethanol and washed in PBS ( $3 \times 5$ min each). Tissues were permeabilized by incubation with PBS-0.25\% Triton $\mathrm{X}-100$ for $15 \mathrm{~min}$ at $4^{\circ} \mathrm{C}$. After washing in PBS $(3 \times 5 \mathrm{~min})$, endogenous peroxidase activity was blocked with $3 \%$ hydrogen peroxide in PBS for 30 min at room temperature (RT). After washing, sections were incubated with PBS-3\% non-fat dry milk in a humid chamber for $1 \mathrm{hr}$ at RT. Then, they were incubated overnight at $4^{\circ} \mathrm{C}$ with the primary antibody goat anti-MMP-2 (1:50/PBS) (sc-6838; Santa Cruz Biotechnologies, Santa Cruz, CA, USA) or rabbit anti-MMP-9 (1:200/ PBS) (Cat. No.444236; Calbiochem, San Diego, CA, USA). For negative controls, the primary antibody was omitted and PBS was added instead. After washing ( $3 \times 5 \mathrm{~min}$ ), sections were incubated for $1 \mathrm{hr}$ at RT with biotinylated rabbit anti-goat secondary antibody (1:400/ PBS) (BA-5000; Vector Laboratories, Burlingame, CA, USA) or biotinylated goat anti-rabbit secondary antibody (1:300/PBS) (BA-1000; Vector Laboratories, Burlingame, CA, USA) for MMP-2 and MMP-9, respectively. After washing, slides were incubated with horseradish peroxidase-conjugated streptavidin (1:400/PBS) (S-911; Molecular Probe Inc. Oregon, USA) for $1 \mathrm{hr}$ at RT. Finally, samples were revealed with 3-3'-diaminobenzidine in chromogen solution (Dab Substrate kit, 957D-22; Cell Marque Rocklin, USA), for 20 min (MMP-9) or 3 min (MMP-2), counterstained with haematoxylin, mounted in DPX, and observed and photographed with an Axiophot Zeiss light microscope (Carl Zeiss, Inc., Oberkochen, Germany). MMP localization and distribution in the different placental zones were analysed.

\section{3 | Protein extraction for western blot}

Total proteins of placental tissues stored at $-70^{\circ} \mathrm{C}$ were first extracted by cutting in ice-cold lysis buffer containing $50 \mathrm{mM}$ Tris- $\mathrm{HCl}(\mathrm{pH} 7.4)$, 1\% NP-40, 1 mM EDTA and $3 \mu$ of protease inhibitor cocktail (Sigma Chemical Co., St. Louis, MO, USA). Tissues were left for 30 min on ice and then homogenized for $1 \mathrm{~min}$. Crude homogenates were centrifuged at $5,500 \mathrm{~g}$ for $15 \mathrm{~min}$ at $4^{\circ} \mathrm{C}$. The supernatant was stored at $-70^{\circ} \mathrm{C}$. Protein content was determined by Bradford assay (Bio-Rad, Hercules, CA, USA).

\subsection{Western blot}

Stored supernatant samples of labyrinth (Lab), marginal haematoma (Hem), non-implantative endometrium (Non-Impl) and endometrium of the implantative zone (Impl-End) were dissolved in sample buffer $(500 \mu \mathrm{M}$ Tris- $\mathrm{HCl}, \mathrm{pH}$ 6.8, 10\% SDS, 30\% glycerol, $0.5 \% \beta$ mercaptoethanol and $0.5 \%$ bromophenol blue), boiled for $5 \mathrm{~min}$ and immediately placed on ice. Equal amounts of proteins $(100 \mathrm{\mu g} / \mathrm{lane})$ under reducing conditions were resolved on $10 \%$ SDS-polyacrylamide gel (SDS-PAGE) (10\% acrylamide/bisacrylamide for resolving gel and $5 \%$ acrylamide/bisacrylamide for stacking gel) in a Mini Protean Cell Bio-Rad system (Bio-Rad). After SDS-PAGE, they were equilibrated in transfer buffer for $15 \mathrm{~min}$ and electrotransferred at $150 \mathrm{~V}$ for $90 \mathrm{~min}$ at constant voltage onto polyvinylidene difluoride membranes (BioRad) using a Mini Trans-Blot Cell (Bio-Rad). Transfer was monitored by Ponceau red staining, and membranes were blocked with $2 \%$ nonfat dry milk in PBS-Tris containing 0.1\% Tween-20 (PBS-T) for 60 min, to minimize non-specific binding of primary antibody.

For MMP-2 detection, goat anti-MMP-2 (1:5 in PBS) antibody was incubated for $72 \mathrm{hr}$ at $4^{\circ} \mathrm{C}$, and after stripping, the rabbit antiactin (Sigma Chemical Co.) (1:2,000 in PBS) was incubated for $24 \mathrm{hr}$ at $4{ }^{\circ} \mathrm{C}$. For MMP-9 detection, the primary antibodies rabbit antiMMP-9 (1:10) (Calbiochem) and rabbit anti-actin (Sigma Chemical Co.) $(1: 2,000)$ were diluted in PBS and incubated for $72 \mathrm{hr}$ at $4^{\circ} \mathrm{C}$. After washing (PBS-T), blots were incubated with the second biotinylated rabbit anti-goat or goat anti-rabbit (Vector Laboratories, Burlingame, CA, USA) (1:1,000/PBS) for $1 \mathrm{hr}$ at RT. After washing (PBS-T), the reaction was enhanced with horseradish peroxidase-conjugated streptavidin (1:1,000) (Molecular Probes) for $1 \mathrm{hr}$, and blots were developed with an enhanced chemiluminescence system using ECL Western blotting system (GE Healthcare) and a bio-imaging analyser (Fujifilm LAS-1000). Pre-stained protein standards (Bio-Rad) with a molecular weight range of approximately 250-25 kDa were used. The bands of MMPs of the placental zones were analysed by densitometric analysis (Image Scn), and optic density was normalized to actins bands.

\section{5 | Gelatin zymography}

Each placental zone was cut and homogenized in lysis buffer [50 mM Tris- $\mathrm{HCl}$ (pH 7.4), $5 \mathrm{mM} \mathrm{CaCl} 2,1 \mu \mathrm{M} \mathrm{ZnCl}_{2}, 1 \%$ Triton X-100], incubated at RT for $2 \mathrm{hr}$ and centrifuged at $13,000 \times \mathrm{g}$ for $15 \mathrm{~min}$ to remove cellular debris. Protein content of supernatant $(100 \mu \mathrm{g})$ was determined according to the Bradford method.

Tissue lysates were mixed with zymogram sample buffer containing $10 \mathrm{mM}$ Tris $-\mathrm{HCl}, 0.06 \%$ bromophenol blue, 10\% glycerol and $2 \%$ SDS. Briefly, gelatin at a final concentration of $1 \mathrm{mg} / \mathrm{ml}$ was incorporated to $10 \%$ sodium dodecyl sulphate-polyacrylamide gel electrophoresis (SDS-PAGE) with 4\% SDS-PAGE stacking. Aliquots from tissue lysate $(20 \mu \mathrm{l}$ approximately) of each sample were loaded and proteins electrophoresed for $1 \mathrm{hr}$ at $100 \mathrm{~V}$. After electrophoresis, gels were washed five times ( $5 \mathrm{~min}$ each) in a Tris-based solution containing $2.5 \%(\mathrm{w} / \mathrm{v})$ Triton $\mathrm{X}-100$ and three times for $10 \mathrm{~min}$ in phosphatebuffered saline (PBS). Then, the gel was incubated in $50 \mathrm{mM}$ Tris- $\mathrm{HCl}$ 
(pH 7.5), $0.15 \mathrm{M} \mathrm{NaCl}$ and $0.005 \mathrm{M} \mathrm{CaCl}_{2}$ at $37^{\circ} \mathrm{C}$ for $48 \mathrm{hr}$. Gels were stained with $0.5 \%(\mathrm{w} / \mathrm{v})$ Coomassie brilliant blue R-250 (Sigma Chem Co.) for $45 \mathrm{~min}$, lightly destained in methanol:acetic acid:water (3:1:6) and finally stored in 5\% (v/v) acetic acid.

Gelatin zymography was photographed with a bio-imaging analyser (Fujifilm LAS-1000). Each gelatinase band was identified by their molecular weight compared with pre-stained protein standards (BioRad) with a range of approximately 250-25 kDa. Enzymatic activities, evaluated as intensity of clear bands in the gel, were performed by image analysis (Image Scn). The different placental zones were run on separate gels with the same amount of protein loaded on each lane. To establish that the gelatinolytic activity observed was due to MMPs, a set of extracts was pre-incubated in the presence of $0.01 \mathrm{M}$ sodium ethylenediaminetetraacetate (EDTA) (Sigma Chemical Co.) before performing electrophoresis, and gels were incubated in the presence of EDTA after electrophoresis; EDTA-dependent disappearance of the corresponding bands confirmed that activity (data not shown). The proteolytic activity appeared as clear bands, visualizing both the proenzyme and the smaller active form, on a blue background. The densitometry of the bands was analysed on digitalized images and assessed by the Image Scn program.

\section{6 | Statistical analysis}

Mean of arbitrary units of MMP expression levels and activity and the $S D$ of samples of placental tissues were analysed by one-way analysis of variance (ANOVA), using Tukey's multiple post-test to determine differences amongst them. Data satisfied assumptions of normality and variance homogeneity required for ANOVA. All statistical analyses were performed with the Instat Program (GraphPAD Software, San Diego, CA, USA). Differences were considered significant when $p<.05$.

\section{3 | RESULTS}

\section{1 | Immunohistochemical detection of MMP-2 and MMP-9 in early placenta}

MMP-2 expression was detected in labyrinthine cytotrophoblast cells and syncytiotrophoblast with a strong staining. Mesenchyme from the CAM presented a weak immunostaining as well as embryonic endothelial cells, while maternal endothelium resulted negative (Fig. 2A). MMP-2 negative control is shown in Fig. 2B. MMP-9 was also immunoexpressed in cytotrophoblast cells and syncytiotrophoblast (Fig. 2C), but with a remarkable weaker intensity than that observed for MMP2. However, the allantoic mesenchyme displayed a very strong immunoreaction to MMP-9, whereas expression in vascular structures was similar to that of MMP-2. MMP-9 negative control is shown in Fig. 2D.

In the endometrium, MMP-2 was expressed in deep glands near to the myometrial tissue, being the expression restricted to the apical side of the epithelium. ECM also presented weak immunoreactivity, revealing MMP-2 secretion (Fig. 3A). Results of rabbit IgG negative control are shown in Fig. 3B. In the superficial glands, MMP-2 was positive in the epithelium of one side of the gland and almost negative in the other side (Fig. 3C). Negative control is shown in Fig. 3D. MMP-9 immunoexpression was also strong in the superficial gland epithelium (Fig. 3E). Negative control is shown in Fig. 3F. Immunohistochemical results are summarized in Table 1.

\section{2 | Placental MMP-2 and MMP-9 expression levels and gelatinolytic activity}

Taken into account the variable MMP immunoexpression in the different cells and tissues of the foetal and maternal canine placenta, the
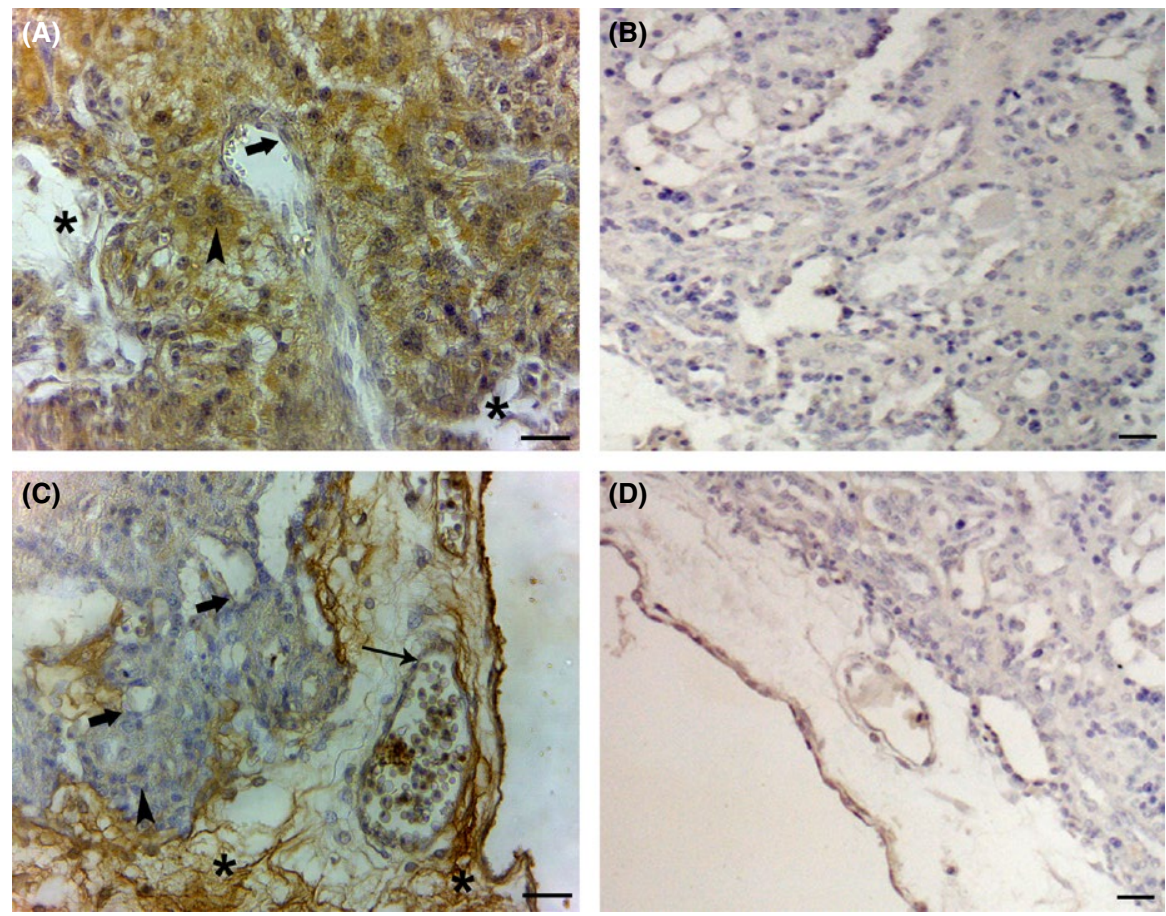

FIGURE 2 Distribution and cellular localization of MMP-2 and MMP-9 immunoexpression in labyrinth of dog early placenta. (A) MMP-2 immunoexpression. Arrowhead points to strongly labelled syncytium. Maternal endothelium was negative (thick arrow). Mesenchyme presented weak immunostaining (asterisks). (B) Negative control for MMP-2 immunoreactivity. (C) MMP-9 immunoexpression. Staining of trophoblast cells was very weak (arrowhead). Thin arrow points to positive embryonic endothelium, whereas thick arrow points to negative maternal endothelium. The mesenchyme presented strong immunoreaction (asterisks). (D) Negative control for MMP-9 immunoreactivity. Scale bar: $20 \mu \mathrm{m}$. IHC, DAB chromogen, haematoxylin counterstain. MMP, matrix metalloproteinases 
FIGURE 3 Distribution and cellular localization of MMP-2 and MMP-9 immunoexpression in maternal zone in dog early placenta. (A) In deep uterine glands near to the myometrium (m), MMP2 expression was positive in the apical side of the epithelium (black arrow). Also the extracellular matrix presented weak immunoreactivity (asterisks), revealing MMP-2 secretion. (B) IgG negative control for MMP-2 in deep uterine glands. (C) In the uterine chambers of superficial glands, MMP-2 was positive in the epithelium of one side of the chamber (thin arrow) and negative in the other (thick arrow). (D) IgG negative control for MMP-2 in superficial glands. (E) MMP-9 immunoexpression was strong in the epithelium of superficial glands (arrows). Also, extracellular MMP-9 immunoreactivity was observed in maternal stroma (asterisks). (F) IgG negative control for MMP-9 in superficial glands. Scale bar: A, B: $20 \mu \mathrm{m}$; C-F: $10 \mu \mathrm{m}$. IHC, DAB chromogen, haematoxylin counterstain. MMP, matrix metalloproteinases
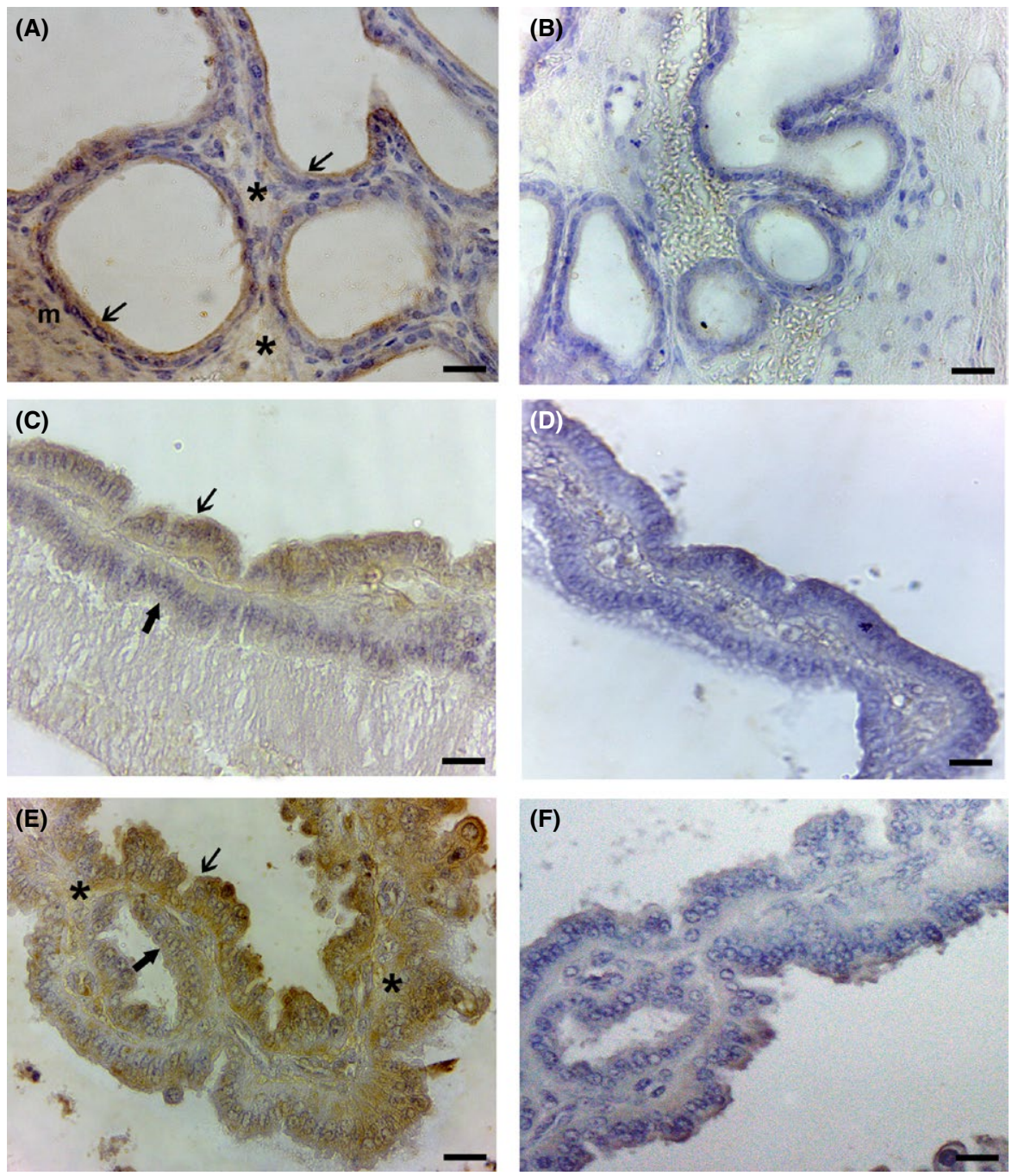

TABLE 1 Summary of localization, distribution and relative expression of MMP-2 and MMP-9 in the dog early placenta

\begin{tabular}{|lcc|}
\hline & MMP-2 & MMP-9 \\
\hline Chorioallantoic membrane & $+/-$ & +++ \\
\hline Mesenchyme & & \\
\hline Labyrinth & +++ & + -- \\
\hline Cytotrophoblast cells & +++ & $+/-$ \\
\hline Syncytiotrophoblast & + & + \\
\hline Embryonic endothelium & - & - \\
\hline Maternal endothelium & & \\
\hline Endometrium & $+/-$ & ++ \\
\hline Junctional zone (epithelium of \\
superficial glands)
\end{tabular}

Immunostaining of MMPs was evaluated in tissues of labyrinth and endometrium and it is summarized as relative intensity as follows: -: negative (without MMP reaction), +/- weakly positive reactivity, ++: moderate reactivity, +++: strong reactivity. MMP, matrix metalloproteinases. interest was, initially, to analyse the whole expression levels of both proteases MMP-2 and MMP-9 in labyrinth, haematoma, implantative endometrium and to compare the non-implantative endometrium of early placenta, by WB semiquantification (Fig. 4).

This analysis showed that all zones of the placenta expressed a band of MMP-2 at 55-60 kDa which corresponded to the active form of the protease (Fig. 4A). The MMP-2 expression level in labyrinth was slightly higher than that in haematoma (Hem) $(p<.05)$, whereas the expression in implantative endometrium (IE) was lower than that in Lab $(p<.01$ ) (Fig. 4A). A band of near $100 \mathrm{kDa}$ of MMP-9 was detected by WB in all the same tissues, but in Lab and Hem the MMP-9 was expressed similarly and in a significant higher level than in the nonimplantative and implantative endometrium ( $p$.001) (Fig. 4B).

Only the active form of MMP-2 $(50 \mathrm{kDa})$ was detected in all maternal and embryonic tissues studied by $\mathrm{Zg}$ (Fig.5A). The activity of MMP-2 was highest in Hem, whereas the activity in non-IE was the lowest. Moreover, MMP-2 activity in the labyrinth was significantly lower than the MMP-2 activity of implantative endometrium $(p<.01)$ (Fig.5A,B). 


\section{(A) MMP-2}
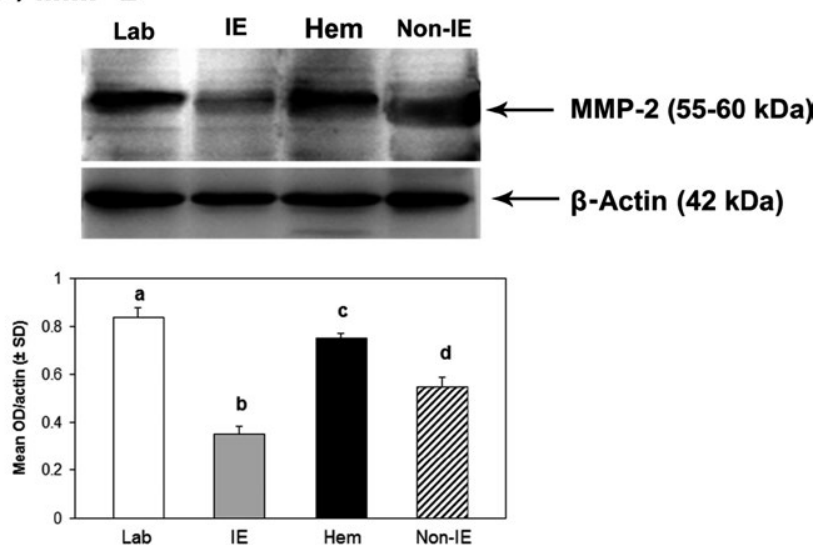

(B) MMP-9
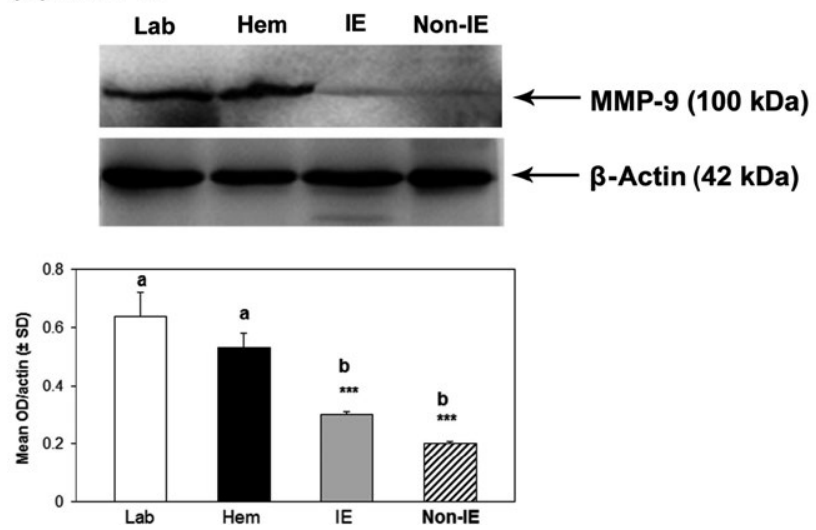

FIGURE 4 Expression levels of MMP-2 and MMP-9 in early dog placenta. (A) Western blot of MMP-2. Upper figure shows representative canine placental tissues showing MMP-2 protein bands according to molecular weight 55-60 kDa of the active form of the MMP-2. Figure below shows the densitometric analysis of MMP-2 protein expression levels normalized to $\beta$-actin, expressed as the mean optic density (OD) of MMP- $2 / \beta$ actin ( $\pm S D$ ). Lab, labyrinth; IE, implantative endometrium; Hem, haematoma; Non-IE, nonimplantative endometrium. This figure shows the most significant increments in MMP-2 expression levels in Lab and Hem, and the lowest expression in the IE and Non-IE. Lab vs IE: $p<.001$; Lab vs Hem: $p<.05$; Lab vs Non-IE: $p<.001$; Hem vs Non-IE: $p<.001$; IE vs Non-IE: $p<.001$ (Tukey's test). Different letters indicate statistical differences. (B) Western blot of MMP-9 Upper figure shows representative areas of the canine placenta showing MMP-9 protein according to antibody for MMP-9, which recognizes both the latent pro-enzyme and the active forms, at MW of 95-110 kDa. It was identified only one band corresponding to MW 100 kDa. Figure below shows the densitometric analysis of MMP-9 expression levels, normalized to $\beta$-actin, as the mean optic density (OD) of MMP-9/ $\beta$ actin $( \pm S D)$. This figure shows the highest MMP-9 expression levels in $\mathrm{Lab}$ and Hem, and the lowest expression in IE and Non-IE. Different letters indicate statistical differences. Lab and Hem vs Non-IE and IE: $p<.001$ (Tukey's test). MMP, matrix metalloproteinases

\section{DISCUSSION}

For several years, it was shown in haemochorial placentae from primates that MMPs play key roles in the processes of implantation
(A)

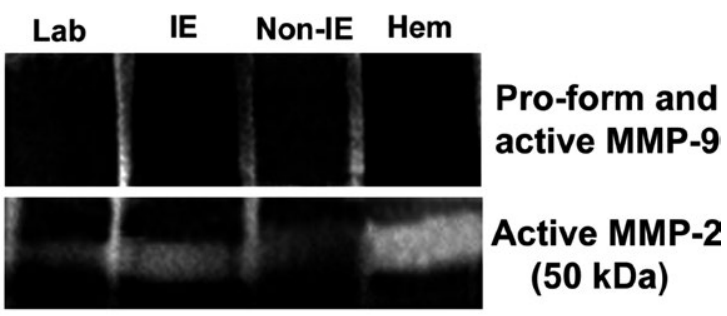

(B)

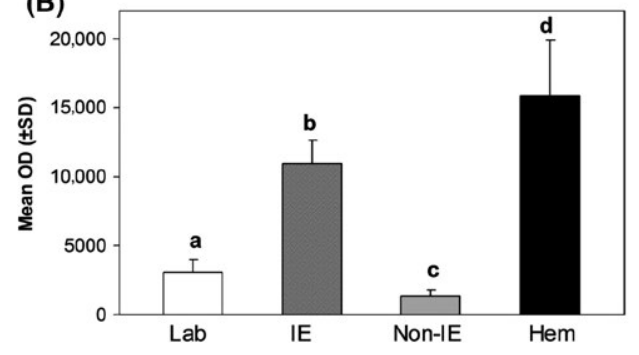

FIGURE 5 MMP-2 and MMP-9 gelatinolytic activities in early canine placenta. (A) Representative MMP-2 and MMP-9 gelatin zymography of samples of placental and maternal tissues of early canine placenta, showing that any form (pro-form or active) of MMP9 was detected at MW range of $110-80 \mathrm{kDa}$, while the same tissues presented activity of the active form of MMP-2 (molecular weight of $50 \mathrm{kDa}$ ). Lane 1: labyrinth; lane 2: implantative endometrium; lane 3: non-implantative endometrium; lane 4: haematoma. (B) Figure shows the activity levels of MMP-2 of placental tissues, expressed as mean optic density (OD) and SD of the active gelatinolytic MMP2 bands. The activity of active MMP- 2 is highest in haematoma (Hem) and significantly moderately increased in the implantative endometrium (IE). Different letters indicate statistical differences. Lab vs IE: $p<.01$; Lab vs Non-IE: $p<.001$; IE vs Non-IE: $p<.001$; IE vs Hem: $p<.05$; Non-IE vs Hem: $p<.001$ (Tukey's test), MMP, matrix metalloproteinases

and early placentation, because of its proteolytic action on various components of the ECM (Li et al., 2014; Wang et al., 2001; $\mathrm{Xu}$ et al., 2000). These enzymes that favour invasiveness processes were also found in TB cells of less studied species (Uekita et al., 2004; Vagnoni et al., 1995, 1998), even in carnivores with endotheliochorial placentas (Beceriklisoy et al., 2007; Walter \& Schönkypl, 2006). The present work confirms the presence of proteases MMP-2 and MMP-9 in the labyrinth, the maternal glands and the marginal haematoma of canine early placenta, together with the activity of MMP-2, mainly in the haematoma. As it was previously observed by Beceriklisoy et al. (2007) in a later gestational state, MMP-2 could be evidenced by IHC in the labyrinth of early canine placenta. MMP-9 immunostaining, on the other hand, was positive in both epithelial and mesenchymal components of the labyrinth, contrary to previous reports in cat (Walter \& Schönkypl, 2006) and in later canine placenta (Beceriklisoy et al., 2007). The MMP-2 and MMP-9 expression extended to the implantative endometrium, including deep glandular areas. According to this, we also demonstrated high MMP-2 and MMP-9 protein expression in labyrinth and implantative endometrium by WB. However, enzymatic activity in the labyrinth, the implantative endometrium and the marginal haematoma was limited to MMP-2. Thus, we find that 
although MMP-9 is well expressed in the early canine placenta, it is not active.

In particular, the activity of the active form of MMP-2 was highest in the haematoma and in the implantative endometrium. The marginal haematoma is the area of major remodelling of tissues, where direct contact occurs between the TB and the maternal blood, due to maternal vessel degradation. This area is considered by some authors (Stoffel, Gille, \& Friess, 1998) as an area of haemochorial placentation within the endotheliochorial placenta. No previous works concerning the activity of MMPs in the canine placental haematoma were found. As marginal haematomas develop during early gestational period (Miglino et al., 2006), we studied this early period of placentation in canine.

Beceriklisoy et al. showed activity of both enzymes in the uterine portion of the placenta and in the labyrinth of later placentas. However, their activity in the labyrinth and in the placental marginal haematoma of the first half of gestation was not clearly established (Beceriklisoy et al., 2007). These results are consistent with those seen in human placenta, where the production of MMP-2 is high in early TBs and decreases in the third trimester, while MMP-9 production increases (Xu et al., 2000). Also, MMP-2 activity is higher than that of MMP-9 at the beginning of gestation (Riley et al., 1999). In the rhesus monkeys (Macaca mulatta), mRNA for MMP-9 was detected on day 18 of pregnancy, but no activity was found at that time, although it did appear later (Wang et al., 2001).

Conversely, in cattle, expression of MMP-9 was very low, both in endometrium and in foetal placenta (Takagi et al., 2007). In the goat placenta, the activity of MMP-2 is moderate in the interplacentomal areas, but varies in the binucleated cells, which fuse with the endometrial epithelium and constitute the most invasive placental component (Uekita et al., 2004). In sheep, the presence of these enzymes in binucleated cells is controversial. Vagnoni et al. (1998) support this idea, while Riley et al. (2000) could not locate them, and proposed that the breakdown of type IV collagen necessary for these cells to migrate and merge with the endometrium depends on paracrine secretion of MMP-2 and MMP-9 by the uninucleate TB cells.

Although MMP-9 activity could not be observed in early canine placenta in any studied area, its activity could come out at late pregnancy, possibly because of the pivotal role of this protease in the angiogenic process (Deryugina \& Quigley, 2006) or in the tissue breakdown at the time of delivery (Yonemoto et al., 2006). In this regard, studies of the junctional zone (the placental releasing site) and of the endometrium in canine preterm placenta are necessary.

Predominance of inactive forms of MMP-2 and MMP-9 in the cat placenta was demonstrated by zymography (Walter \& Schönkypl, 2006). The lower activity of MMP-2 in cats compared with levels reported in dogs might be associated with the increased invasiveness in the placenta of the latter, and related to the occurrence of the disease known as subinvolution of the placentation sites in the bitches (Fernández, Portiansky, Barbeito, \& Gimeno, 1998). It has not been studied yet whether MMP-2 and MMP-9 are involved in the pathogenesis of this disease.
In our work in early canine placenta, the presence of the proteases MMP-2 and MMP-9 was demonstrated in the labyrinth as in the maternal tissues, besides in marginal haematoma. However, the enzymatic activity of these proteases was limited to MMP-2.

Altogether, our results, added to those of other researchers, indicate that while both MMPs have similar structures and substrates, their expression and activity in the placenta vary according to the tissue and gestational time, suggesting different involvement at specific times of placentation, in different groups of animals.

Changes in the activity of MMPs throughout pregnancy appear to be associated with the processes of invasiveness. MMPs of maternal tissues, in different species (Wang et al., 2001), may act on both endometrial basal lamina and TB tissue, leading to the loss of the epithelial cell contact with the basement membrane. The relevance of the study of these enzymes of ECM resides in their role in the processes of invasiveness, maternal-foetal angiogenesis, therefore in the establishment of a correct foetal nutrition. In humans, a decline in the production of MMP-2 and MMP-9 was associated with growth retardation (Merchant et al., 2004). On the other hand, some authors (Kutzler et al., 2012) have argued that the canine placenta could serve as a model for study of human pre-eclampsia, considering the high invasive capacity of its cells, the fundamental role of MMPs for invasiveness and the altered expression of these enzymes during pre-eclampsia (Wang et al., 2010). Given the importance of the metalloprotease expression and activation, specific tissue and gestational period, it is necessary to perform further studies to establish the location, MMPs expression and activity of canine placenta throughout pregnancy.

\section{ACKNOWLEDGEMENT}

The authors acknowledge $\mathrm{Ht}$ Ruben Mario for his histotechnical assistance.

\section{FUNDING SOURCES}

The authors disclosed receipt of the following financial support for the research, authorship and publication of this article: BIDPICT-2008-2210 from Agencia Nacional de Promoción Científica y Tecnológica, Argentina (EC-grant), and grants from UNLP V/229 research project, Argentina.

\section{CONFLICT OF INTERESTS}

None of the authors have any conflict of interest to declare.

\section{AUTHOR CONTRIBUTIONS}

$\mathrm{CB}, \mathrm{EC}$ and $\mathrm{MD}$ designed the research; $\mathrm{MD}, \mathrm{MV}, \mathrm{RH}, \mathrm{CS}$ and $\mathrm{LC}$ were involved in sampling; $\mathrm{CB}, \mathrm{EC}, \mathrm{MD}, \mathrm{MV}$ and $\mathrm{RH}$ performed analysis and interpretation of data; $M D, C B$ and $E C$ drafted the manuscript; $C B$, $\mathrm{EC}, \mathrm{MD}, \mathrm{MV}, \mathrm{RH}, \mathrm{CS}$ and $\mathrm{LC}$ revised the manuscript; $\mathrm{MD}, \mathrm{CB}$ and $\mathrm{EC}$ answered reviewers. 


\section{REFERENCES}

Aralla, M., Groppetti, D., Caldarini, L., Cremonesi, F., \& Arrighi, S. (2013). Morphological evaluation of the placenta and fetal membranes during canine pregnancy from early implantation to term. Research in Veterinary Science, 95, 15-22.

Barbeito, C. G., Fernández, P. E., Gimeno, E. J., \& Portiansky, E. L. (2004). Immunohistochemical and morphometric study of the decidual cells in the domestic cat placenta. Biocell, 28, 229.

Barrau, M. D., Abel, J. H., Torbit, C. A., \& Tietz, W. J. (1975). Development of the implantation chamber in the pregnant bitch. The American Journal of Anatomy, 143, 115-130.

Beceriklisoy, H. B., Walter, I., Schäfer-Somi, S., Miller, I., Kanca, H., Izgür, H., \& Aslan, S. (2007). Matrix metalloproteinase (MMP)-2 and MMP-9 activity in the canine uterus before and during placentation. Reproduction in Domestic Animals, 42, 654-659.

Conrad, M. L., Freitag, N., Diessler, M. E., Hernandez, R., Barrientos, G., Rose, M., Casas, L. A., Barbeito, C. G., \& Blois, S. M. (2016). Differential spatiotemporal patterns of galectin expression are a hallmark of endotheliochorial placentation. American Journal of Reproductive Immunology, 75, 317-325.

Cross, J. C., Werb, Z., \& Fisher, S. J. (1994). Implantation and the placenta: key pieces of the development puzzle. Science, 266, 1508-1518.

Deryugina, E. I., \& Quigley, J. P. (2006). Matrix metalloproteinases and tumor metastasis. Cancer and Metastasis Reviews, 25, 9-34.

Enders, A. C., \& Carter, A. M. (2012a). Review: The evolving placenta: Different developmental paths to a hemochorial relationship. Placenta, 33(Suppl), S592-S598.

Enders, A. C., \& Carter, A. M. (2012b). The evolving placenta: Convergent evolution of variations in the endotheliochorial relationship. Placenta, 33, 319-326.

Evans, H. E., \& Sack, W. O. (1973). Prenatal development of domestic and laboratory mammals: Growth curves, external features and selected references. Zentralblatt für Veterinärmedizin, 2(1), 11-45.

Fernández, P. E., Barbeito, C. G., Portiansky, E. L., \& Gimeno, E. J. (2000). Intermediate filament proteins expression and sugar moieties of the normal canine placenta. Histology and Histopathology, 15, 1-6.

Fernández, P. E., Diessler, M. E., Pachame, A., Ortega, H. H., Gimeno, E. J., Portiansky, E. L., \& Barbeito, C. G. (2014). Intermediate filament proteins expression and carbohydrate moieties in trophoblast and decidual cells of mature cat placenta. Reproduction in Domestic Animals, 49, 263-269.

Fernández, P. E., Portiansky, E. L., Barbeito, C. G., \& Gimeno, E. J. (1998). Characterization of cytotrophoblastic-like cells present in subinvolutioned placental sites of the bitch. Histology and Histopathology, 13 995-1000.

Gram, A., Hoffmann, B., Boos, A., \& Kowalewski, M. P. (2015). Expression and localization of vascular endothelial growth factor A (VEGFA) and its two receptors (VEGFR1/FLT1 and VEGFR2/FLK1/KDR) in the canine corpus luteum and utero-placental compartments during pregnancy and at normal and induced parturition. General and Comparative Endocrinology, 223, 54-65.

Kautz, E., de Carvalho Papa, P., Reichler, I. M., Gram, A., Boos, A., \& Kowalewski, M. P. (2015). In vitro decidualisation of canine uterine stromal cells. Reproductive Biology and Endocrinology, 13, 85. doi:10.1186/ s12958-015-0066-4

Kutzler, M., Sahlfeld, L., \& Fellows, E. (2012). Who let the dogs in: A canine trophoblast invasion model for pre-eclampsia. Reproduction in Domestic Animals, 47(Suppl 6), 186-189.

Li, C. M., Hou, L., Zhang, H., \& Zhang, W.Y. (2014). CCL17 Induces Trophoblast Migration and Invasion by Regulating Matrix Metalloproteinase and Integrin Expression in Human First-Trimester Placenta. Reproductive Sciences, 1-13, doi: 10.1177/1933719113519170.

McGrath, K., \& Palis, J. (2005). Hematopoiesis in the yolk sac: More than meets the eye. Experimental Hematology, 33, 1021-1028.
Merchant, S. J., Crocker, I. P., Baker, P. N., Tansinda, D., Davidge, S. T., \& Guilbert, L. J. (2004). Matrix metalloproteinase release from placental explants of pregnancies complicated by intrauterine growth restriction. Journal of the Society for Gynecologic Investigation, 11, 97-103.

Miglino, M. A., Ambrósio, C. E., Dos Santos Martins, D., Wenceslau, C. V., Pfarrer, C., \& Leiser, R. (2006). The carnivore pregnancy: The development of the embryo and fetal membranes. Theriogenology, 66, 1699-1702.

Nothdurft, W., Braasch, E., Calvo, W., Prummer, O., Carbonell, F., Grilli, G., \& Fliedner, T. M. (1984). Ontogeny of the granulocyte/macrophage progenitor cell (GM-CFC) pools in the Beagle. Journal of Embryology and Experimental Morphology, 80, 87-103.

Novaro, V., Pustovrh, C., Colman-Lerner, A., Radisky, D., LoNostro, F. Paz, D., ... González, E. (2002). Nitricoxide induces gelatinase A (matrix metalloproteinase 2) during rat embryo implantation. Fertility and Sterility, 78, 1278-1287.

Pfarrer, C., Winther, H., Leiser, R., \& Dantzer, V. (1999). The development of the endotheliochorial mink placenta: Light microscopy and scanning electron microscopical morphometry of maternal vascular casts. Anatomy and Embryology, 199, 63-74.

Pieri, N., Souza, A. F., Casals, J. B., Roballo, K., Ambrósio, C. E., \& Martins, D. S. (2015). Comparative development of embryonic age by organogenesis in domestic dogs and cats. Reproduction in Domestic Animals, 50, 625-631.

Riley, S. C., Leask, R., Chard, T., Wathen, N. C., Calder, A. A., \& Howe, D. C. (1999). Secretion of matrix metalloproteinase-2, matrix metalloproteinase-9 and tissue inhibitor of metalloproteinases into the intrauterine compartments during early pregnancy. Molecular Human Reproduction, $5,376-381$

Riley, S. C., Webb, C. J., Leask, R., McCaig, F. M., \& Howe, D. C. (2000), Involvement of matrix metalloproteinases 2 and 9, tissue inhibitor of metalloproteinases and apoptosis in tissue remodelling in the sheep placenta. Journal of Reproduction and Fertility, 118, 19-27.

Staun-Ram, E., Goldman, S, Gabarin, D, \& Shalev, E. (2004). Expression and importance of matrix metalloproteinases 2 and 9 (MMP-2 and MMP-9) in human trophoblast invasion. Reproductive Biology and Endocrinology, 2(1), 59.

Sternlicht, M. D., \& Werb, Z. (2001). How matrix metalloproteinases regulate cell behaviour. Annual Review of Cell and Developmental Biology, 17, 463-516.

Stoffel, M. H., Gille, U., \& Friess, A. E. (1998). Scanning electron microscopy of the canine placenta. Italian Journal of Anatomy and Embryology, 103, 291-300.

Takagi, M., Yamamoto, D., Ohtani, M., \& Miyamoto, A. (2007). Quantitative analysis of messenger RNA expression of matrix metalloproteinases(MMP-2 and MMP-9), tissue inhibitor-2 of matrix metalloproteinases (TIMP-2), and steroidogenic enzymes in bovine placentomes during gestation and postpartum. Molecular Reproduction and Development, 74, 801-807.

Teesalu, T., Masson, R., Basset, P., Blasi, F., \& Talarico, D. (1999). Expression of matrix metalloproteinases during murine chorioallantoic placenta maturation. Developmental Dynamics, 214, 248-258.

Uekita, T., Yamanouchi, K., Sato, H., Tojo, H., Seiki, M., \& Tachi, C. (2004). Expression and localization of matrix metalloproteinases (MT1-MMP, MMP-2) and tissue inhibitor of metalloproteinase-2 (TIMP-2) during synepitheliochorial placentation of goats (Capra hircus). Placenta, 25, 810-819.

Vagnoni, K. E., Ginther, O. J., \& Lunn, D. P. (1995). Metalloproteinase activity has a role in equine chorionic girdle cell invasion. Biology of Reproduction, 53, 800-805.

Vagnoni, K. E., Zheng, J., \& Magness, R. R. (1998). Matrix metalloproteinases-2 and -9 , and tissue inhibitor of metalloproteinases-1 of the sheep placenta during the last third of gestation. Placenta, 19, 447-455.

Verstegen-Onclin, K., \& Verstegen, J. (2008). Endocrinology of pregnancy in the dog: A review. Theriogenology, 70, 291-299.

Walter, I., \& Boos, A. (2001). Matrix metalloproteinases (MMP-2 and MMP9) and tissue inhibitor-2 of matrix metalloproteinases (TIMP-2) in the 
placenta and interplacental uterine wall in normal cows and in cattle with retention of fetal membranes. Placenta, 22, 473-483.

Walter, I., \& Schönkypl, S. (2006). Extracellular matrix components and matrix degrading enzymes in the feline placenta during gestation. Placenta, 27, 291-306.

Wang, H., Li, Q., Shao, L., \& Zhu, C. (2001). Expression of matrix metalloproteinase-2, -9,-14, and tissue inhibitors of metalloproteinase-1, -2, -3 in the endometrium and placenta of rhesus monkey (Macaca mulatta) during early pregnancy. Biology of Reproduction, 65, 31-40.

Wang, Z., Lu, S., Liu, C., Zhao, B., Pei, K., Tian, L., \& Ma, X. (2010). Expressional and epigenetic alterations of placental matrix metalloproteinase 9 in preeclampsia. Gynecological Endocrinology, 26, 96-102.
Wooding, P., \& Burton, G. (2008). Comparative placentation: Structures, functions and evolution (pp. 169-181). Berlin: Springer-Verlag.

Xu, P., Wang, Y., Zhu, S., Luo, S., Piao, Y., \& Zhuang, L. (2000). Expression of matrix metalloproteinase-2, -9 and -14 , tissue inhibitors of metalloproteinase-I, and matrix proteins in human placenta during the first trimester. Biology of Reproduction, 62, 988-994.

Yonemoto, H., Young, C. B., Ross, J. T., Guilbert, L. L., Fairclough, R. J., \& Olson, D. M. (2006). Changes in matrix metalloproteinase (MMP)-2 and MMP-9 in the fetal amnion and chorion during gestation and at term and preterm labor. Placenta, 27, 669-677. 